\title{
21 Tesla MRI Microscopy of Mice Kidney, Heart and Skin: Quantitation of MRI Visible Features \\ S. SHARMA and R. SHARMA,
}

${ }^{1}$ National High Magnet Field Lab, Florida State University, Tallahassee, Florida, United States of America, ${ }^{2}$ Department of Electrical Engineering, FAMU-FSU College of Engineering, Tallahassee, Florida, United States of America

\section{$\underline{\text { Abstract }}$}

Excised mice kidney, heart and skin MRI visible features were quantitated using ex vivo 21 Tesla MRI microimager. The fast MRI acquisition protocols of FLASH, gradient echo flow compensated (GEFC) techniques were used to visualize microvasculature of mice kidney, mice heart including cardiac arteries, veins, cordate tendons and mice skin epidermis with hair follicle features.

Object

To acquire images in 10 seconds to visualize the features of mice kidney, heart and skin tissue by 21 Tesla MRI and compare skin MRM images with histology. Introduction

Bruker-Biospin FLASH and GEFC imaging protocols are unique to acquire 3D images within 15 seconds. 21Tesla MRI microimaging offers details of tissue organization at cellular level with measurement accuracy up to 15 microns [1]. Other significant capability of MRI microimaging is to speculate tissue biochemical details by using superparamagnetic nanoparticles [2]. Glycolipids in skin, myoglobin in heart and lipoproteins in kidney tissues play significant role and are likely MRI visible quickly by MRI microscopy. However, the measurement accuracy and its interpretation remain controversial in biochemical NMR microscopy [2]. The utility of bound superparamagnetic iron-oxide particles with antimetabolite such as anti-troponin or myoglobin remains to investigate [1]. In presence of high magnetic field, multiple contrast approach visualizes distribution of tissue metabolites with specific signal intensities by T1-wt/T2-wt/proton density weighted images. Bruker Biospin PARAVISION multislice-multiecho technique is unique to generate the MRM contrast [2]. Recently, 21 Tesla MRM at National High Magnetic Field Laboratory has enhanced the possibility of 3D visualization of rat skin, mice brain, kidney and lungs with their vasculature.

Materials and Methods

The fresh excised skin, heart and kidney tissues from mice were imaged at 21 Tesla MRI using 3D FLASH, 3D GEFC within 15 seconds and multislice multiecho (MSME) techniques on PARAVISION 3.2 standard techniques (Bruker Biospin, Bellarica) to acquire T1-, T2- and proton density high resolution images. To standardize and quantify the MR signal intensity at different tissue locations, glycolipid, myoglobin and lipoprotein solutions were imaged as phantoms as earlier [1]. To confirm the composition and measurement accuracy, excised mice skin, heart and kidney samples were processed for histology sections to coregister digital images with MRM images. Skin epidermis, cardiac ventricles, aorta, auricles, coronary and blood vessels, renal cortex and microvilli area were measured by planimetry.

Results

The T1 weighted MR signal intensities of glycolipid, lipoprotein phantoms in capillary was higher than T2 weighted signal (Figure 1). The excised heart tissue showed mice heart ventricles, auricles and 80-100 micron thick coronary territories were distinct at 21 Tesla (Figure 2). Main features of 21 Tesla MRM were: 1 . The signalnoise-ratio and resolution were high with spatial resolution of 20 microns; 2. 3D FLASH images were acquired in 10 seconds; 3. The 3D GEFC images showed noise on background but acquisition time was less than 15 seconds; 4. MSME images showed better structural details of skin(epidermis 100 micron, hair thickness 50 micron); 5 . The main features of heart right and left chambers with distinct 3-4 layers of myocyte arrays, septum, tricuspid and mitral valves were distinct; 6 . The kidney cortex, renal artery and appendix were distinct as attached with kidneys(Figure 3); 7.The mice hair with hair follicle, epidermis, and dermis layers were distinct and measurable (Figure 4). However, the limitations of 21 Tesla MRM were observed for long time experiments due to less stable gradients, coil $B_{0}$ inhomogeneity and noise as main determinants during 3D experiments.

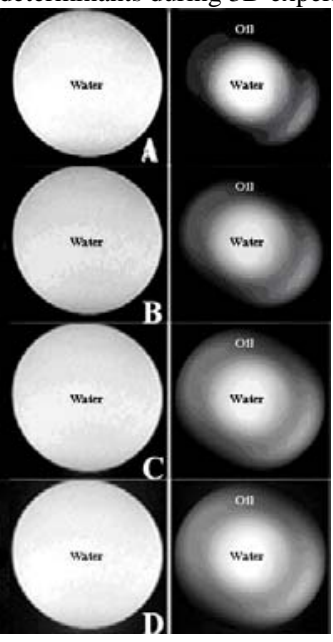

Fig 1: Glycopilipid phantom T1 images(A,B,C,D 5-20mM) Outer tube lipid, inner tube of water show different $\mathrm{T}_{2}$ images

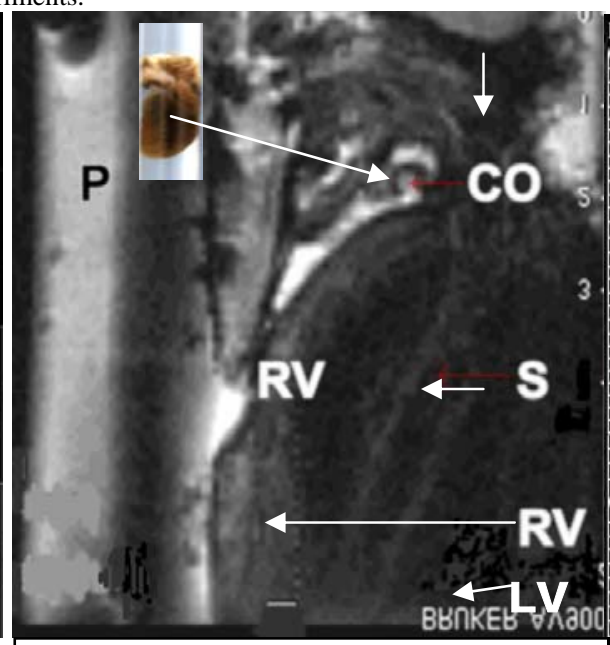

Fig 2: An ex vivo mice heart FLASH image in 8 sec. The image at $\mathrm{TE}=5 \mathrm{~ms}, \mathrm{TR}=750 \mathrm{~ms}$, shows coronary (CO), right \& left ventricle(LV/RV),A-V septum(S) at $21 \mathrm{~T}$ resolution 15 micron with Phantom(P)
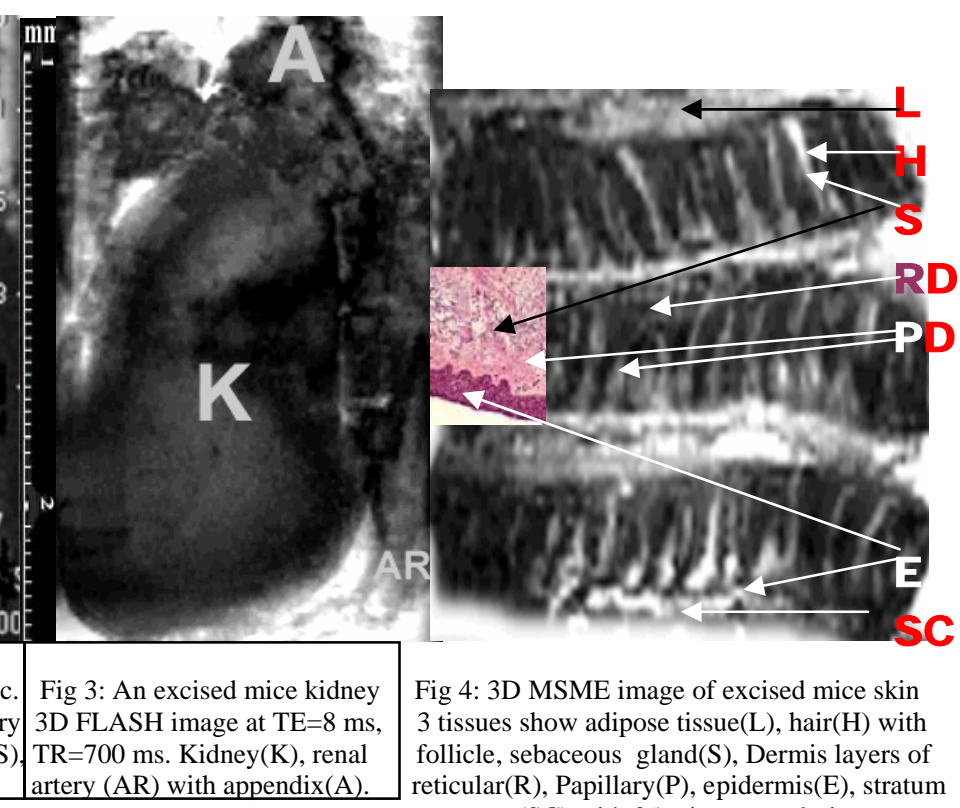

Fig 4: 3D MSME image of excised mice skin 3 tissues show adipose tissue( $\mathrm{L})$, hair(H) with follicle, sebaceous gland(S), Dermis layers of reticular(R), Papillary(P), epidermis(E), stratum corneum(SC) with 35 micron resolution.

\section{Discussion}

The ultrahigh resolution NMR microscopy is new research tool with lot of promise with superior spatial resolution up to 20 micron level visualization of microvasculature in mice heart and kidney tissue in both in vivo or ex vivo experiments. By use of 3D FLASH, microdissected anatomy and NMR microscopy may serve as promising tool of rapid therapeutic monitoring. Other great promise of cell trafficking by use of nanotechnology may provide as smart biosensor approach using them for NMR microscopy. However, the possibility of such cell and molecular imaging remains uncertain today. Recently our focus was pharmacodynamic imaging of adverse toxic effect of jet fuels across rat skin by using high resolution multicontrast approach [3]. The sensitivity of NMR microscopy is shown up to 35 micron thick hair enveloped by hair follicle 80 micron thick in Figure 4. The mice coronary artery 100 micron thickness is distinct in Figure 2. 3D visualization is other benefit of 21 Tesla MRM experiments. However, ultrahigh magnetic field has own demerits which are not fully known. Our experience showed frequently that SNR and noise were highly dependent on gradient stability, $\mathrm{B}_{0}$ inhomogeneity and susceptibility effects.

Conclusion

At 21 Tesla, the mice kidney and heart showed better resolution and high SNR with distinct renal and cardiac morphology with vasculature. The histology matched MRM images showed the power of microimaging to predict the microdetails of different skin features The enhanced ultrahigh resolution 21T MRI microimaging may be useful molecular imaging and functional monitoring research tool.

References

1. Sharma R et al. J Expt Nano Sci. 1(1), 2006 \& NTSI 2006 \# 905; 